\title{
Alteration of the Electron Density of the Lower Ionosphere With Ground-Based Transmitters
}

\author{
Pietro P. Lombardini \\ Moore School of Electrical Engineering, University of Pennsylvania, Philadelphia, Pa.
}

(Received May 15, 1964; revised July 28, 1964)

\begin{abstract}
Using computational techniques as described by Carleton and Megill, and Megill to calculate the difference between rate of attachment of electrons to $\mathrm{O}_{2}$ and the ionization rate in air as a function of electric field, the power required to create breakdown in the ionosphere has been calculated. These calculations are compared with extrapolated microwave breakdown data and found to agree satisfactorily.

The propagation of the primary beam is altered by the electrons created by it. This gives origin to a nonlinear problem, which is very difficult to deal with in the general case. However, a steady state approximate solution has been found, considering diffusion in one dimension. The solution is applied to the design of a possible atmospheric experiment.
\end{abstract}

\section{Introduction}

The possibility of altering the characteristics of the lower ionosphere utilizing ground-based transmitters has been considered by several investigators. In particular, studies have been made concerning the achievement of a decrease in electron density [Molmud, 1964], an increase in the electron collision frequency [Farley, 1963] and the excitation of air glow [Bailey, 1959 and Megill, 1964]. Little attention, however, has been given to utilizing groundbased transmitters to achieve an increase of electron density. The aim of this paper is to investigate the parameters involved in obtaining such an increase.

The first step of the present work involves the definition of breakdown as created by a high-frequency electromagnetic wave in a boundless, slightly ionized homogeneous medium. Experimental data for these conditions of breakdown are not available; however, the electric field necessary to initiate breakdown (breakdown threshold) may be obtained in either of the following two ways. One way consists in performing direct laboratory measurements of breakdown in finite enclosures, and then scaling the results to the open air case. The other way consists of computing the electron distribution function in the mixture of gases considered $\left(\mathrm{N}_{2}, \mathrm{O}_{2}\right.$, and $\left.\mathrm{O}\right)$ and in the presence of the electric field. This distribution function computed by utilizing the experimental data for the various cross sections involved is in turn used to evalute the electron attachment and ionization frequencies (i.e., $\nu_{a}$ and $\nu_{i}$ respectively). The breakdown threshold, $E_{b}$ (defined as that field for whicn $\nu_{i}=v_{a}$ ) may therefore be calculated.

The breakdown threshold is shown to decrease for certain altitudes at a faster rate than that specified hy the inverse of the distance law which applies to VHF waves in the far field of the transmitter. From this, it is concluded that breakdown can be achieved if the proper power is available. Furthermore, it is shown that once breakdown is obtained, the steady state electron density is critically dependent upon the difference of the altitude rate of changes of the breakdown field and the inverse of the distance field.

Because of this dependence on the altitude rate of change of the exciting field, greater electron densities may be achieved using a sufficiently large antenna such that breakdown occurs in the near field.

A characterization of the attachment-ionization process is shown to indicate an altitude for which the optimum breakdown efficiency exists.

\section{Characterization of the Breakdown Threshold Electric Field}

A VHF radiowave traveling upward in the $D$ or in the lower $E$ region is assumed to be idealized as a plane wave propagating in a weakly ionized, quasihomogeneous plasma. The breakdown conditions applicable may therefore be identified with those pertaining to an electric field of constant orientation and amplitude in a weakly ionized air. In general, the breakdown condition of an electric discharge is defined by the electric field (breakdown threshold, $E_{b}$ ) for which equilibrium is achieved between the gain of electrons due to ionizing collisions, and the loss due to the various mechanisms which remove electrons from the discharge region (e.g., attachment, recombination and diffusion). Because of the absence of walls, and the weak ionization for the case considered, the breakdown is predominantly controlled by attachment, while diffusion has a negligible role. Conditions of this kind are rarely encountered 
in the laboratory where the walls confining the discharge play a considerable role in removing electrons. Brown and Rose [1957] have shown that the experimental data of microwave breakdown threshold in air can be characterized by a curve of $\frac{E_{\text {eff }}}{p}$ versus the product $p L$, where $E_{\text {eff }}$ is the "effective" breakdown electric field defined by the formula,

$$
E_{\mathrm{eff}}=E_{b} \frac{\nu}{\sqrt{\nu^{2}+\omega^{2}}}
$$

where,

$$
\begin{aligned}
& \nu=\text { average electron collision frequency } \\
& \omega=\text { radian frequency of wave } \\
& p=\text { air pressure } \\
& L=\text { breakdown gap spacing. }
\end{aligned}
$$

This curve flattens out for increasing values of $p L$ approaching the asymptotic value of $3 \times 10^{3} \mathrm{~V} / \mathrm{m}$ $\mathrm{x}(\mathrm{mm}$ of $\mathrm{Hg})$. Introducing this value into (1), considering a collision frequency as a function of pressure given by $5.3 \times 10^{9} p(\mathrm{c} / \mathrm{s})$ [MacDonald, Gaskell, and Gitterman, 1963], one obtains the following simple equation relating the rms value of the breakdown threshold field in air with the pressure,

$$
E_{b}=3 \times 10^{3} p \sqrt{1+\left(\frac{\omega}{5.3 \times 10^{9} p}\right)^{2}} .
$$

Specification of breakdown conditions in open space may also be obtained utilizing a different approach. That is, the energy distribution function of the electrons under the influence of an oscillating electric field in a mixture of $\mathrm{N}_{2}, \mathrm{O}_{2}$, and $\mathrm{O}$ may be calculated. In this approach, the experimental data introduced are the collision cross sections of the electrons with the above mentioned components of air. Carleton and Megill [1962], have given a detailed analysis of the problem of determining the electron energy distribution function under these conditions. Megill has constructed a computer program for the IBM 704 of Raytheon, which gives the electron energy distribution function, $f(u)$, for the interval of electron energy, $u$, from 0.01 to $20.0 \mathrm{eV}$, and having neutral particle, density, temperature, geomagnetic field, frequency, and the electric field intensity as parameters. The solution applies to a situation in which the total number of electrons does not change. Providing that the rate of change of the total number of electrons is small, and incorporating perturbation techniques, the solution $f(u)$ may be used for evaluating the rate of increase of electrons due to ionizing collisions.

$$
\nu_{i}=\frac{\sum_{i} N_{i} \int_{0}^{\infty} u f(u) \sigma_{i I}(u) d u}{\left(\frac{m}{2}\right)^{1 / 2} \int_{0}^{\infty} u^{1 / 2} f(u) d u}
$$

or the rate of decrease of electrons due to attachment,

$$
\nu_{a}=\frac{\sum_{i} N_{i} \int_{0}^{\infty} u f(u) \sigma_{i A}(u) d u}{\left(\frac{m}{2}\right)^{1 / 2} \int_{0}^{\infty} u^{1 / 2} f(u) d u}
$$

where $N_{i}, \sigma_{i I}$, and $\sigma_{i A}$ are the concentration, ionization cross section and attachment cross section for the $i$ th component of air considered (i.e., $\mathrm{N}_{2}, \mathrm{O}_{2}$, and $\mathrm{O}$ ) and $m$ is the electron mass. In the actual computations, the only attachment phenomenon considered is the dissociative attachment, $\mathrm{O}_{2}+e \rightarrow \mathrm{O}+\mathrm{O}^{-}$. Ionization and attachment collision frequency obtained in this way may be characterized as a function of the electric field intensity $E$, holding all other parameters constant. $\quad \nu_{a}$ grows rapidly for moderate fields and quickly tends to saturate, while $\nu_{i}$ starts a quick rise at higher field intensities. The crossing of the two curves $\left(\nu_{i}=\nu_{a}\right)$ corresponds to the breakdown threshold $E_{b}$. Using the atmospheric model presented in the Handbook for Astronautical Engineering [Koelle, 1961] extensive computations have been made for the following choice of parameters: $f=50 \mathrm{Mc} / \mathrm{s}$, geomagnetic field $=5 \times 10^{-5} \mathrm{~Wb} / \mathrm{m}^{2}$, and concentrations and temperatures corresponding to the height interval, 55 to $105 \mathrm{~km}$. The corresponding values of $E_{b}$ as a function of altitude are shown in figure 1 by the solid curve. In the same figure, the dotted curve shows the $E_{b}$ altitude variation as obtained using (2). The good agreement between the two methods of approach is apparent from this figure. The collision frequencies given by (3) and (4) are required for evaluating the rate of change of the total number of electrons given by,

$$
\frac{\partial N}{\partial t}=\left(\nu_{i}-v_{a}\right) N
$$

Computed values of $\nu_{i}-\nu_{a}$ are plotted in figure 2 as a function of the excess of electric field, $\Delta E$, and the same range of parameters previously specified for figure 1 . The relationship between $\nu_{i}-\nu_{a}$ and $\frac{\Delta E}{E_{b}}$ is approximately linear.

In figure $3, \nu_{i}-\nu_{a}$ is plotted versus height for constant electric field above threshold breakdown. This figure shows that there exists an altitude at which the creation of new electrons occurs with a maximum efficiency. This optimum height corresponds to the condition for which the average collision frequency approximately equals the frequency of the electric field; i.e.,

$$
\nu\left(h, E_{b}\right) \simeq f .
$$

Figure 4 shows the relationship between frequency and altitude as obtained by (6), using for $\nu$, MacDonald, Gaskell, and Gitterman [1963] approximation. To each of these frequencies corresponds a breakdown threshold electric field, which may be determined using (2). 


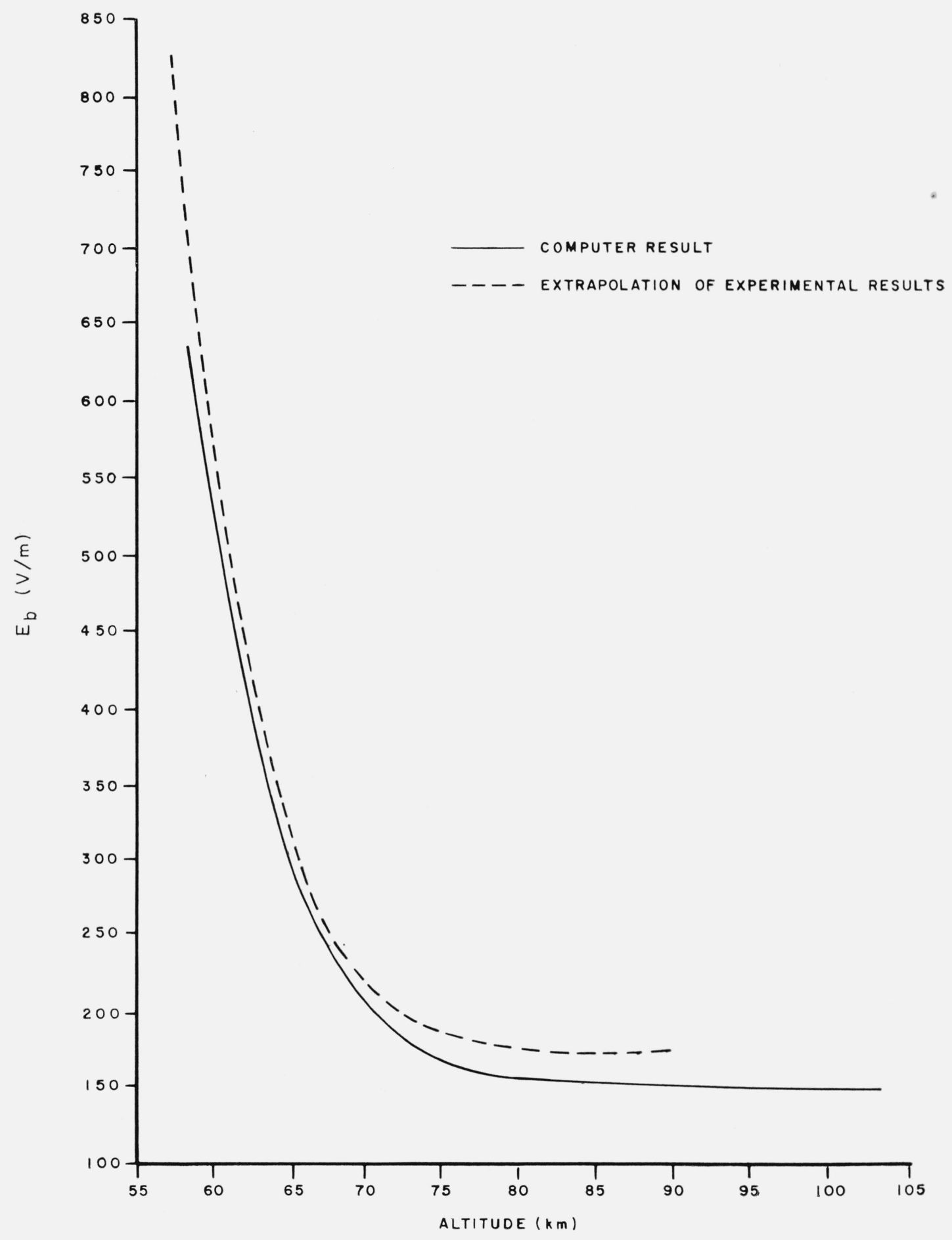

Figure 1. Breakdown threshold electric field versus altitude for a frequency of $50 \mathrm{Mc} / \mathrm{s}$. 


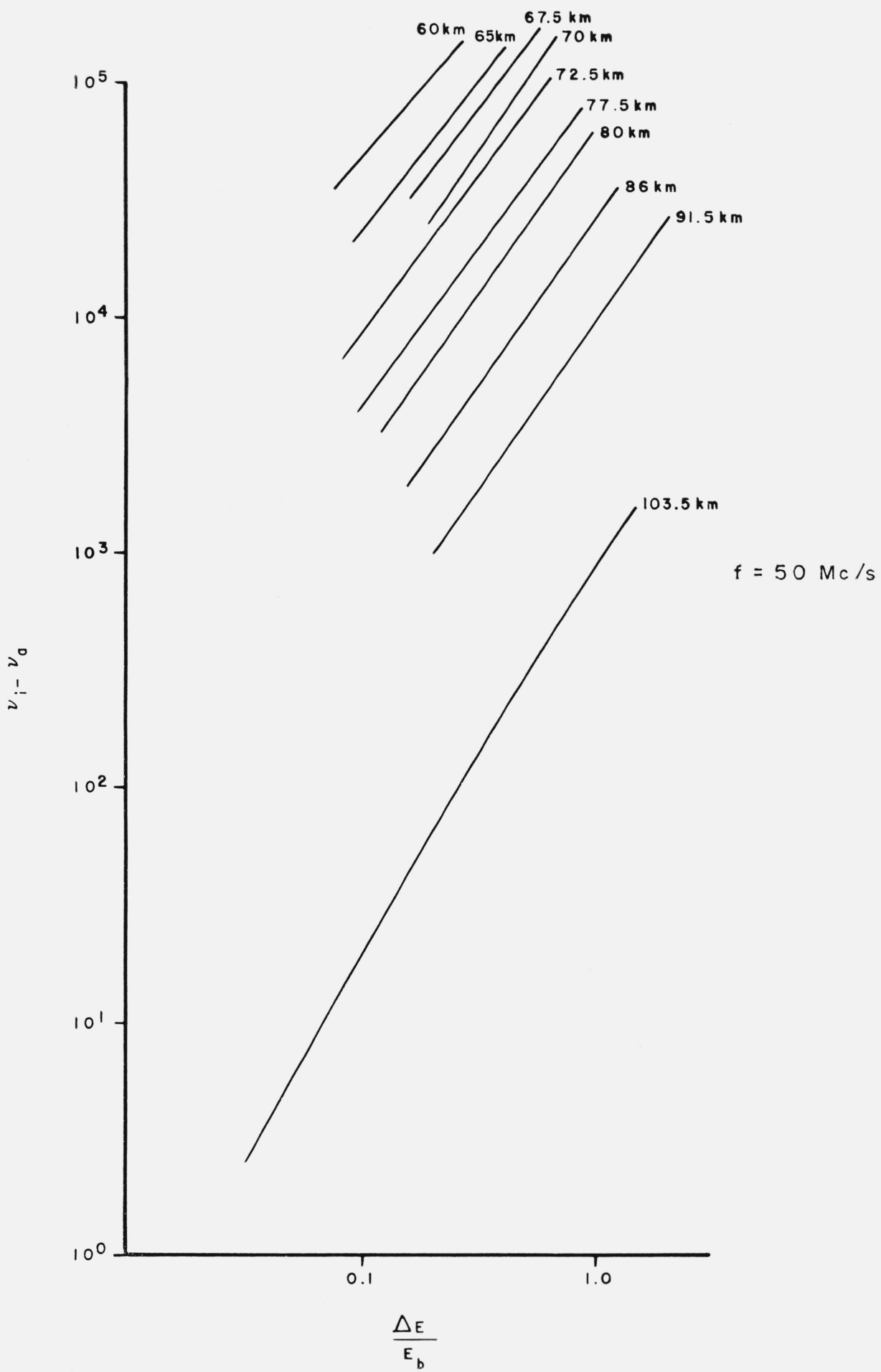

FIGURE 2. The difference of the ionization and attachment collision frequencies as a function of the percent increase of excess electric field above breakdown. 


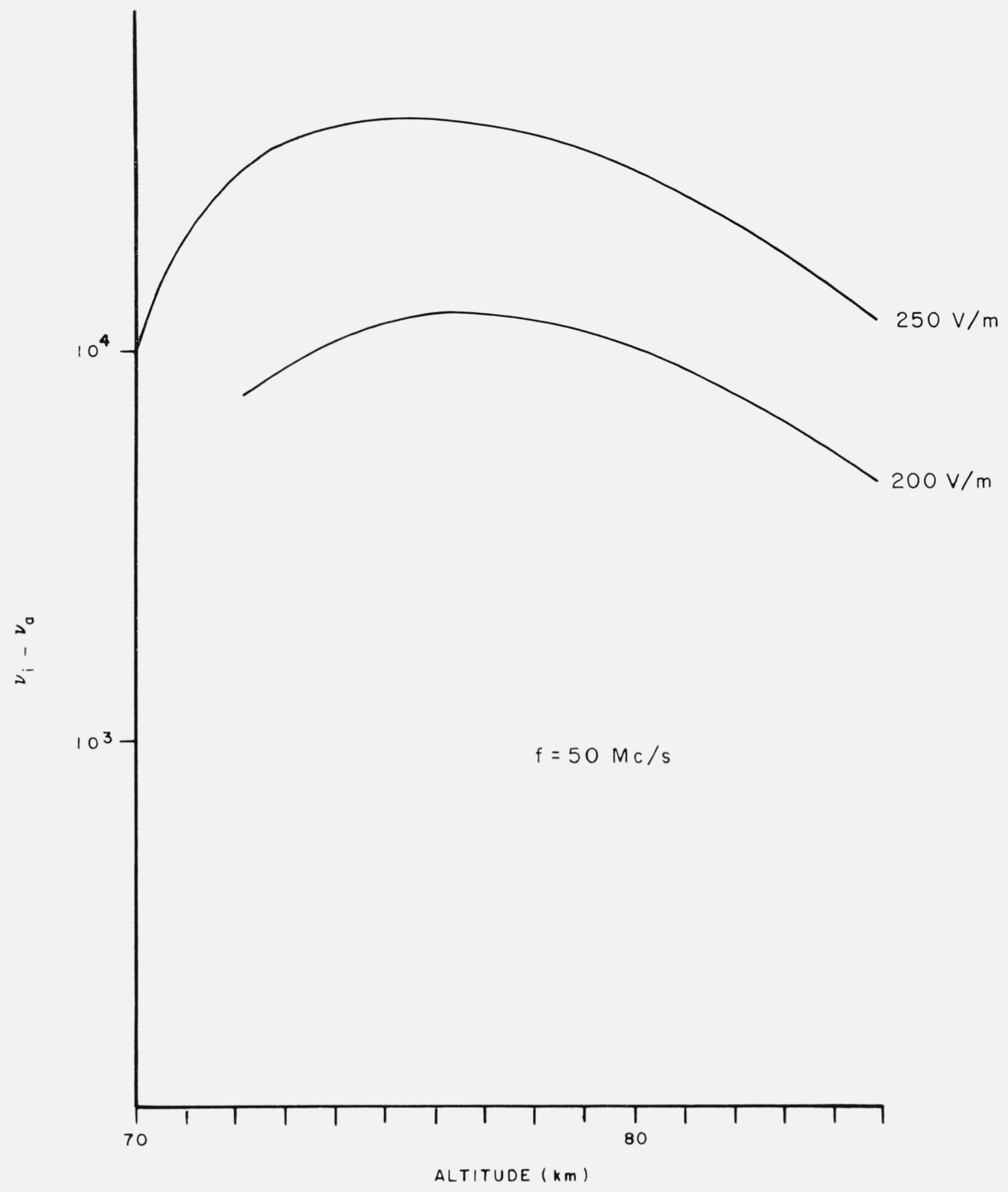

FIGURE 3. The difference of the ionization and attachment collision frequencies as a function of altitude for the field strengths. indicated.

\section{Maximum Attainable Increase of Electron Density}

In the previous section, the conditions required for obtaining breakdown have been examined. The problem is now that of determining the maximum electron density which may be achieved using radio waves. For this purpose, consider a VHF wave propagating vertically upward. Under normal ionospheric conditions, a wave in this band of frequencies is negligibly absorbed by the ionosphere. However, when the electric field strength of the wave becomes larger than the breakdown threshold, the electron density will increase and hence the wave may suffer an exponential attenuation. In free space, the electric field of the wave may be expressed as:

$$
E(x)=E_{0} \Phi(x)
$$

where $E_{0}$ is the amplitude of field intensity at an altitude, $h_{0}$, from the ground, where the source is assumed to be located. Here, $x$ is the local vertical coordinate in a system whose origin is at a height $h_{0}$, and $\Phi(x)$ represents the functional variation in the absence of absorption. In the "far field" of an antenna,

$$
\Phi(x)=\frac{1}{1+\frac{x}{h_{0}}} .
$$




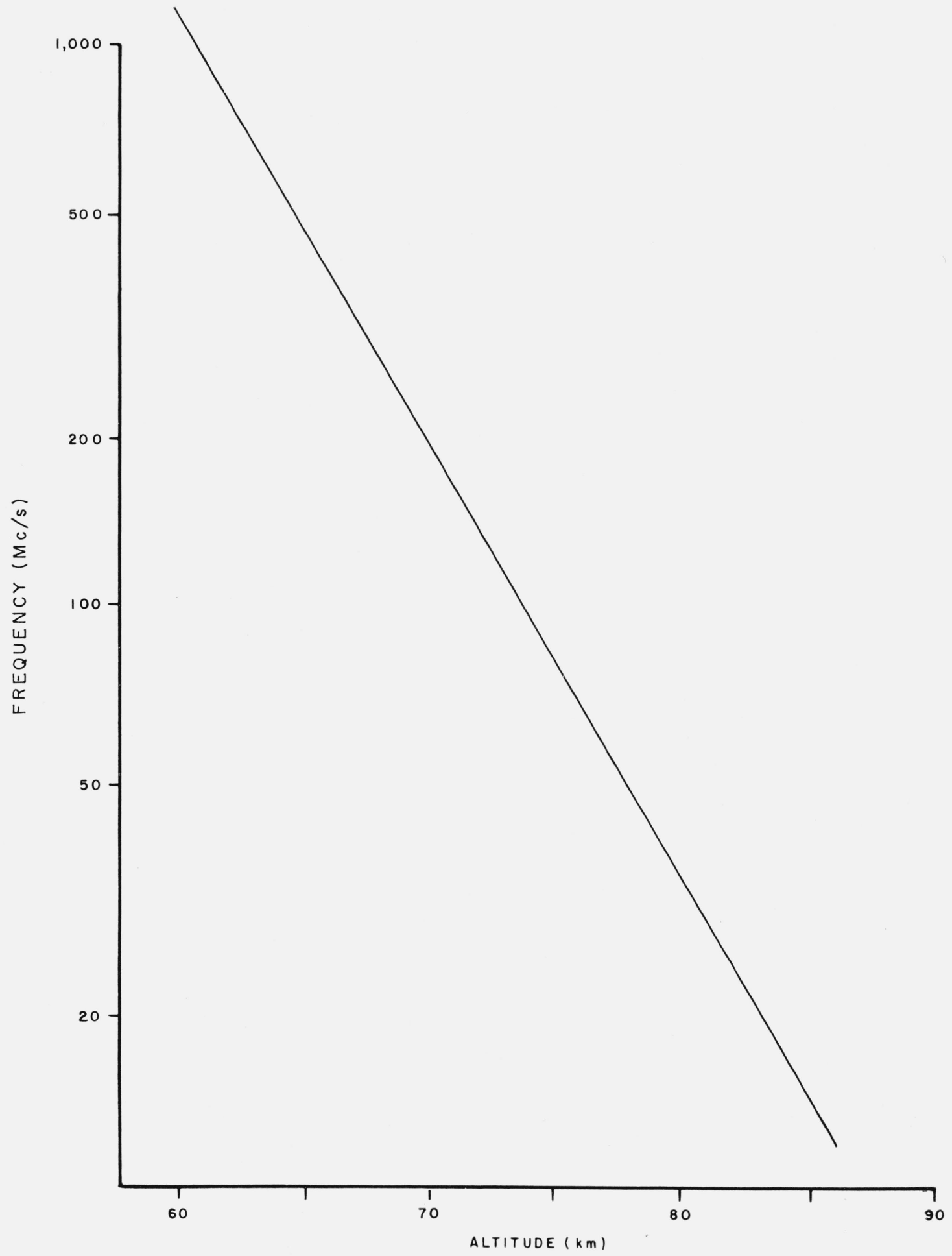

FIGURE 4. Frequency of maximum ionization efficiency versus altitude.

88 
Along the axis of a spherical antenna excited in phase and focused at $h_{0}, \Phi(x)$ is given by,

$$
\Phi(x)=\frac{\sin \left(\frac{\pi D^{2}}{8 \lambda h_{0}^{2}} x\right)}{\left(\frac{\pi D^{2}}{8 \lambda h_{0}^{2}} x\right)}
$$

where $D$ is the antenna diameter and $\lambda$ the wavelength.

If the propagation takes place in ionized air, the wave is subjected to exponential absorption. When the electron density is sufficiently low, the differential power absorbed along the wave path is,

$$
\frac{d}{d x}\left[\frac{E^{2}(x)}{\eta_{0}}\right]=-N(x) \frac{\frac{2}{3} \frac{(e E(x))^{2}}{m} \int_{0}^{\infty} u^{3 / 2} \alpha(u) \frac{\partial f(u)}{\partial u} d u}{\int_{0}^{\infty} u^{1 / 2} f(u) d u}
$$

where $N(x)$ is the electron density at the altitude $h_{0}+x$ and $\alpha(u)$ is given by,

$$
\alpha(u)=\frac{\nu}{\nu^{2}+\omega^{2}}
$$

disregarding the earth's magnetic field. Proceeding to integrate (10) in the interval $x=0$ to the arbitrary distance $x$,

$$
E(x)=E_{0} \Phi(x) \exp \left[-\int_{0}^{x} A(E, x) N(x) d x\right]
$$

where the quantity $A(E, x)$ is a slowly varying function of $E$ and $x$.

In order to simplify the problem, let us for the moment disregard diffusion. Assume that for the radio waves propagating upward with some spatial variation, $\phi(x)$ (e.g., inverse of the distance law) breakdown conditions are reached at the altitude $h_{1}$. The physical situation may be explained from figure 5 where the spatial variation of the electric field of the wave and the breakdown threshold

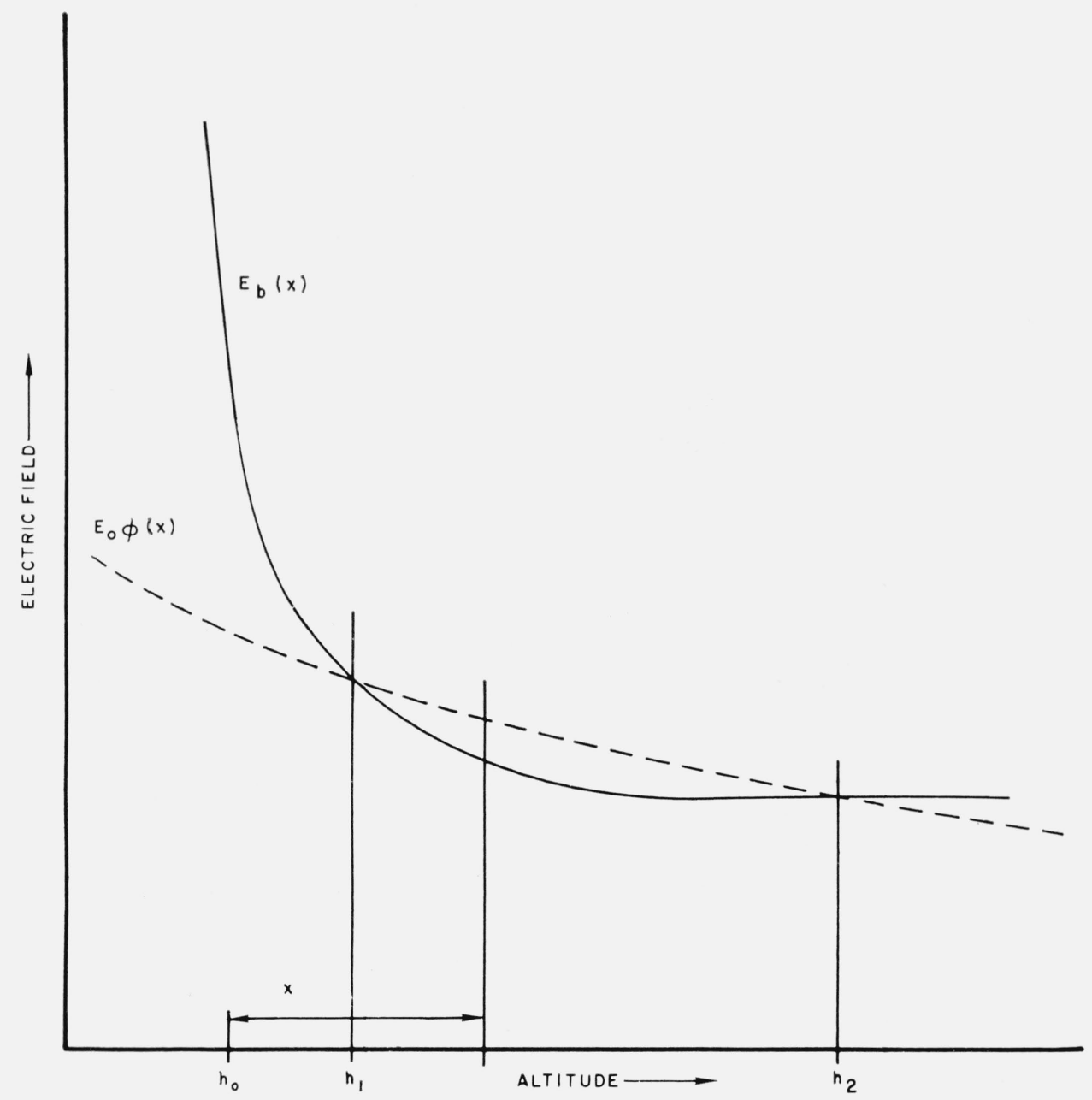

FIGURE 5. Sketch of the variation of the free space field strength (dashed line), and of the breakdown threshold (solid line) versus altitude. 
variation are plotted as a function of altitude. These two curves cross at two altitudes, $h_{1}$ and $h_{2}$. Consider a point located at the altitude $h_{1}+x$, where the spatial variation field curve lies above the breakdown curve. Here the intensity of the wave will change with time because of the attenuation due to the time increasing electron density. Hence, assume the spatial variation curve of figure 5 represents the established situation at time, $t=0$. It follows at this point $\left(h_{1}+x\right)$ that the electron density will start increasing exponentially with time according to the solution of (5),

$$
N(x, t)=N_{0} \exp \left[\left(\nu_{i}-v_{a}\right) t\right]
$$

where $N_{0}$ is the previously existing electron density. It has already been pointed out that the quantity $\left(\nu_{i}-v_{a}\right)$ is critically dependent upon the quantity $\Delta E=E-E_{b}$ (see fig. 2). Substituting (13) into (12),

$$
\begin{aligned}
& \Delta E=E_{0} \Phi(x) \exp \\
& {\left[-\int_{0}^{x} A(x) N_{0} \exp \left[\left(\nu_{i}-\nu_{a}\right) t\right] d x\right]-E_{b}(x) . }
\end{aligned}
$$

Equation (14) does not take into account any mechanism limiting the growth of electron density as a function of time, such as losses due to diffusion or recombination. Therefore, for increasingly large times, satisfaction of (14) requires that $\Delta E$ approaches zero. In the limit condition,

$$
\begin{array}{r}
\operatorname{Lim}_{t \rightarrow \infty} \int_{0}^{x} A(E, x) N(x, t) d x=-\ln \frac{E_{b}(x)}{E_{0} \phi(x)} \\
N_{\max }=\operatorname{Lim}_{t \rightarrow \infty} N(x, t)=\frac{1}{A(E, x)}\left[\frac{\frac{d \phi(x)}{d x}-\frac{d E_{b}(x)}{d x}}{E_{b}(x)}\right] .
\end{array}
$$

An important observation of (16) is that the steady state distribution of $N$ is critically dependent upon the slopes of the field and the breakdown threshold curves.

\section{Diffusion Problem}

Equation (16) was obtained ignoring diffusion. The rigorous solution taking diffusion into account is difficult. However, for steady state, if the electric field and the density of the neutral components of air are slowly varying functions of the coordinates $x$, the electron balance may be approximated by the equation:

$$
D(E, x) \frac{d^{2} N(x)}{d x^{2}}=-N(x)\left(\nu_{i}-\nu_{a}\right)
$$

where

$$
D(E, x)=\frac{\int_{0}^{\infty} u^{3 / 2} \nu^{-1}(u) f(u) d u}{\frac{3}{2} m \int_{0}^{\infty} u^{1 / 2} f(u) d u}
$$

is the diffusion coefficient which is assumed to be a slowly varying function of the altitude and the electric field.

Equations (12) and (17) describe fairly well the steady state situation for $N(x)$ and $E(x)$. The system can be solved using the boundary conditions suggested by physical considerations (see fig 6). The boundary conditions will thus be chosen at two altitudes $h_{1}$ and $h_{2}$, one below and the other above the crossing of the breakdown threshold. For this altitude, the value of $N$ and $\frac{d N}{d x}$ are assumed to be known. Examining (12) and (17) we note that while $A(E, x)$ and $D(E, x)$ are slowly varying functions of $E$ and $x$, the function $\nu_{i}-v_{a}$ may undergo large changes for small changes of either variable (as shown in fig. 6). Thus, (17) is very sensitive to small changes of $E$. This renders the system of (12) and (17) highly nonlinear and difficult to deal with. This system has been solved numerically by Albertoni, Bocchieri, and Daneri [1963] with the help of an IBM 7090 computer.

\section{Numerical Examples}

Two cases are considered here which employ the results of the previous section. The first case deals with the "far field" of the transmitter. The parameters selected are,

$$
\begin{gathered}
f=50 \mathrm{Mc} / \mathrm{s} \\
h_{1}=71 \mathrm{~km} \\
E_{b}=196 \mathrm{~V} / \mathrm{m}
\end{gathered}
$$

In figure 7 is plotted the electron density distribution as obtained using (13) (curve on the right). Also plotted (curves on the left; note the change in scale) are the solutions of the system of (12) and (17). The two curves, which correspond to the different "trial" parameters chosen in the numerical integration, represent the physical solution as long as they coincide. Beyond the point of bifurcation they fail to indicate a physical solution. The region of coincidence could be prolonged with a better choice of parameters. However, the data in figure 8 shows that beyond the region considered, the approximation obtained neglecting diffusion (13) is adequate.

The second case refers to a hypothetical transmitting antenna large enough such that the breakdown field is located at its focus. Here it is assumed that the antenna is a spherical basin whose surface is radiating in phase. The law of variation of the field intensity near the focus is thus assumed to be 


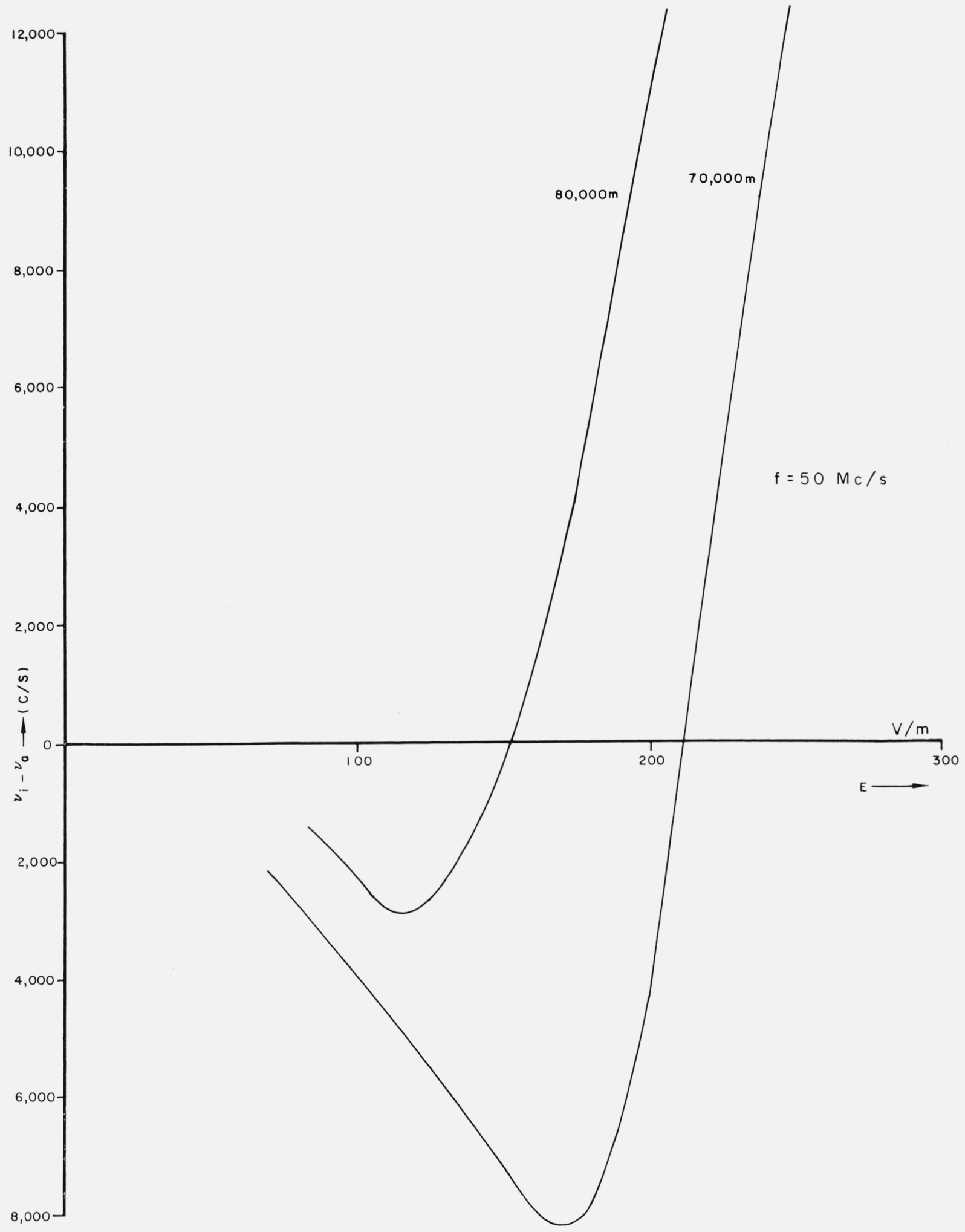

FIGURE 6. Variation of the difference of the ionization and attachment collision frequencies as a function of the field strength. 


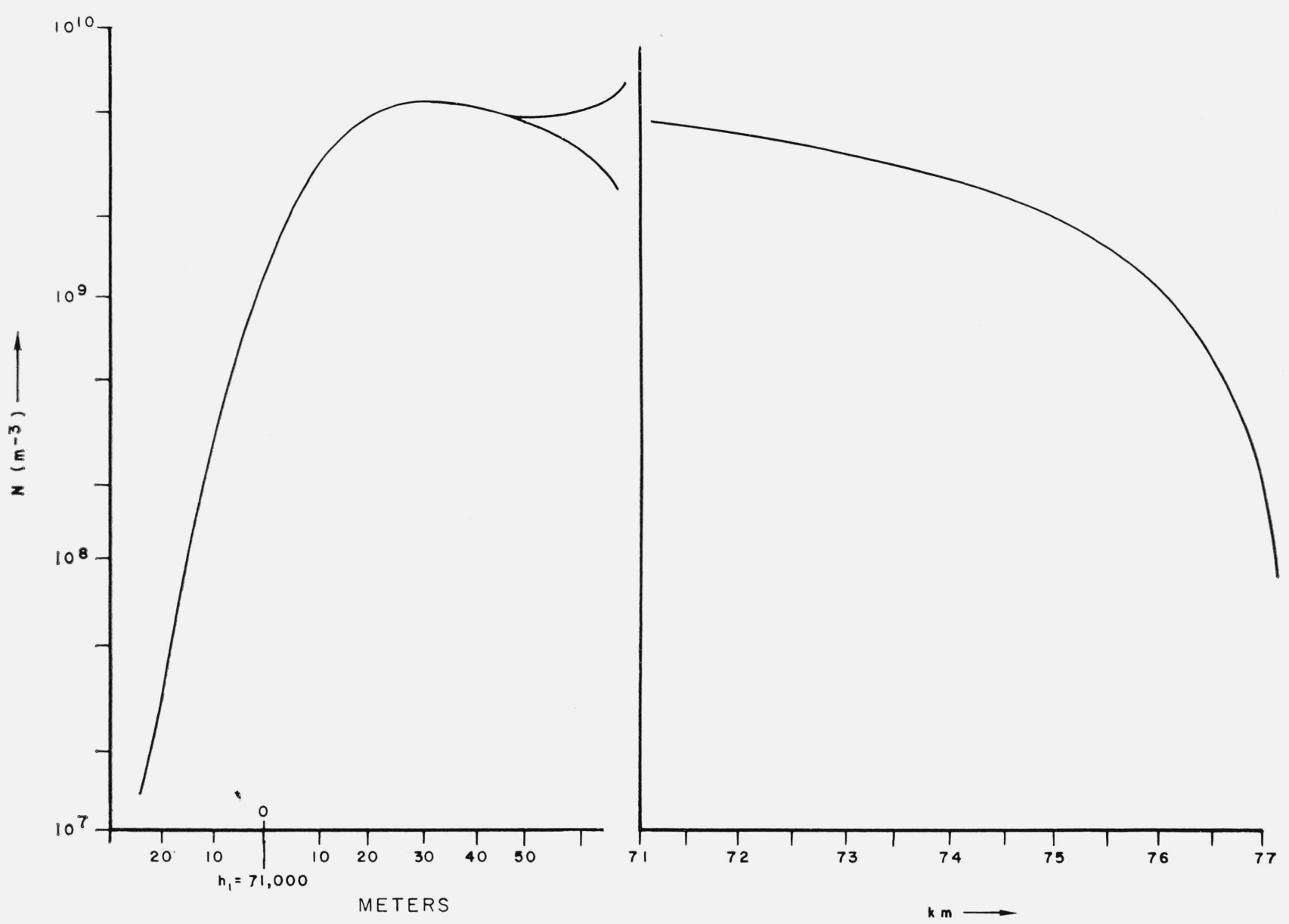

Figure 7. Electron density distribution for case $1\left[\mathrm{f}=50 \mathrm{Mc} / \mathrm{s} \mathrm{E}_{\mathrm{b}}=196 \mathrm{~V} / \mathrm{m}\right.$, far field of transmitter $]$.

given by (9) where,

$$
\frac{\pi D^{2}}{8 \lambda h_{1}^{2}}=1.7 \times 10^{-2} / \mathrm{m}
$$

The other parameters are chosen such that in absence of electronic absorption, the electric field radiated reaches a maximum of $200 \mathrm{~V} / \mathrm{m}$ at the altitude of 78 $\mathrm{km}$, where the focus of the antenna is assumed to be located. In figure 8 is plotted the electron density variation with altitude obtained using (16). Also plotted are the solution of the system of (12) and (17) which is valid as a physical solution up to the point of bifurcation.

\section{Power Requirements}

No mention has been made yet concerning the power required for achieving breakdown. The ground-based upward radiating transmitter needed for obtaining the previously mentioned breakdown fields must have a power-gain product $P G$ which for free wave propagation is given by:

$$
P G=4 \pi h^{2} E_{b}^{2} / \eta_{0}
$$

where $\eta_{0}$ is the wave impedance in space.

The power $P$ fed into the antenna is determined once the gain $G$ of the antenna is known. For the case in which the ionization takes place in the far field of the transmitting antenna, the antenna dimensions are limited by the condition:

$$
h \geq 2 D^{2} / \lambda \text {. }
$$

Assuming a paraboloid antenna of diameter $D$, the maximum antenna gain can not exceed the limiting value:

$$
G_{\max }=\pi^{2} h / 2 \lambda .
$$

On the other hand, when the ionization takes place in the near field of the antenna, and the antenna is focused, the value of the gain is only limited by practical factors like construction accuracy and cost.

In table 1 are tabulated breakdown transmitter parameters for the conditions of altitude and frequency given by figure 4 . For the far field case the maximum gain in the table is obtained using formula (21). For the near field case, the assumed gain at focus in the table is based on gains achieved by far field antennas for the same frequency. 


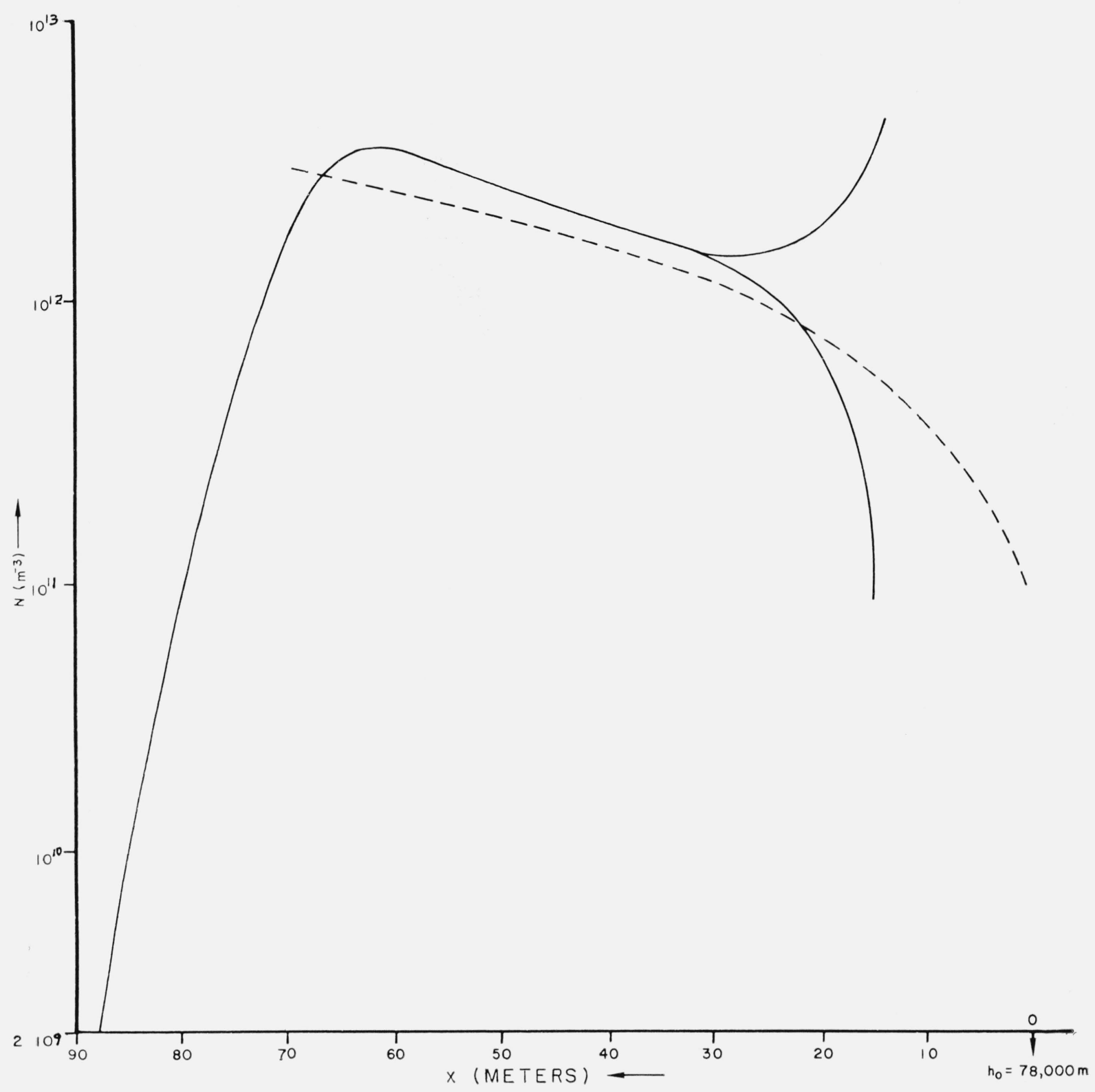

Figure 8. Electron density distribution for case $2\left[\mathrm{f}=50 \mathrm{Mc} / \mathrm{s}, \mathrm{E}\left(\mathrm{h}_{1}\right)=200 \mathrm{~V} / \mathrm{M}, \mathrm{E}_{\mathrm{b}}=156 \mathrm{~V} / \mathrm{m}\right.$, near field of transmitter $]$.

TABLE 1

\begin{tabular}{|c|c|c|c|c|c|c|}
\hline \multirow{2}{*}{$\begin{array}{l}\text { Break- } \\
\text { down } \\
\text { altitude }\end{array}$} & \multirow{2}{*}{$\begin{array}{l}\text { Frequency } \\
\text { for } \\
\text { optimum } \\
\text { ionization } \\
\text { efficiency }\end{array}$} & \multirow{2}{*}{$\begin{array}{l}\text { Power-gain } \\
\text { product } \\
\text { PG }\end{array}$} & \multicolumn{2}{|c|}{ Far field case } & \multicolumn{2}{|c|}{ Near field case } \\
\hline & & & $\underset{\text { gain }}{\text { Maximum }}$ & Power & $\begin{array}{l}\text { Assumed } \\
\text { gain } \\
\text { at focus }\end{array}$ & Power \\
\hline $\mathrm{km}$ & $\mathrm{Mc} / \mathrm{s}$ & Watts & & Megawatt & & Megawatt \\
\hline 60 & 1,000 & 1. $2 \quad 10^{15}$ & 1.0 & 1,200 & 7.5 & 160 \\
\hline 65 & 500 & $3.8 \quad 10^{14}$ & & 690 & 3. 7 & 100 \\
\hline 70 & 250 & 1. $0 \quad 10^{14}$ & 2.9 & 340 & 1. 9 & 53 \\
\hline 75 & 100 & 2. $0 \quad 10^{13}$ & 1. 3 & 160 & 7.5 & 18 \\
\hline 80 & 40 & $3.5 \quad 10^{12}$ & 5.3 & 66 & $3.0 \quad 10^{5}$ & 12 \\
\hline
\end{tabular}

Note that the powers listed above are relative to the condition needed for starting breakdown. Therefore, in order to obtain an increase in electron density, the power required should be larger than the ones tabulated according to the square of the ratio of the field used to the field needed for starting breakdown at square of the ratio

\section{Conclusions}

It has been shown that a radio wave radiated by a ground-based transmitter may achieve breakdown in the $D$ or lower $E$ ionospheric regions. For each altitude, a frequency exists at which there corresponds an optimum ionization efficiency. The maximum electron density which can be obtained critically depends upon the altitude rate of change of both the breakdown field and the free space transmitted field strength. This characteristic automatically limits the maximum obtainable electron density which may be achieved in the far field of an antenna. This limitation may, however, be overcome by having breakdown take place in the near field. 
The study described in this paper has been totally supported by the Raytheon Company, for which the author is a Consultant. Acknowledgement should also be given to all the people who have worked on this project: L. R. Megill (National Bureau of Standards, Boulder, Colo.), S. Albertoni, P. Bocchieri, A. Daneri (Applications of Scientific Research, Milan, Italy), C. F. Eaton, R. C. Gunther (Raytheon Company).

\section{References}

Albertoni, S., P. Bocchieri, and A. Daneri (1963), Conference on applications of electronic computers to space problems, Florence, 12-13 September.

Bailey, V. A. (1959), Some possible effects caused by strong gyrowaves in the ionosphere, J. Atmospheric Terrest. Phys. 14, 299.

Brown, S. C., and D. Rose (1957), Microwave gas discharge breakdown in air, nitrogen and oxygen, J. Appl. Phys. 28, 561
Carleton, N. P., and L. R. Megill (1962), Electron energy distribution in slightly ionized air under the influence of electric and magnetic fields, Phys. Rev. 126, 2089-2099.

Farley, D. T. Jr. (1963), Artificial heating of the electrons in the $F$-region of the ionosphere, J. Geophys. Res. 68, 401.

Koelle, H. H., ed. (1961), Handbook of astronautical engineering (McGraw-Hill Book Co., New York, N.Y.).

MacDonald, A. D., D. U. Gaskell, and H. N. Gitterman (1963), Microwave breakdown in air, oxygen, and nitrogen, Phys. Rev. 130, 1841-1850.

Megill, L. R. (1964), The excitation of optical radiation by high power density radio beams, presented at the Conference on Nonlinear Processes in the Ionosphere, Dec. 16-17, 1963 at Boulder, Colo., Techn. Note 211, 5, 69-86.

Molmud, P. (1964), Use of radio transmitters to decrease $D$-region electron density, presented at the Conference on Nonlinear Processes in the Ionosphere, Dec. 16-17, 1963 at Boulder, Colo., Techn. Note 211, 6, 1-88.

(Paper 69D1-445) 